\title{
Intrinsic GTPase activity of K-RAS monitored by native mass spectrometry
}

Zahra Moghadamchargari ${ }^{1}$, Jamison Huddleston ${ }^{1}$, Mehdi Shirzadeh ${ }^{1}$, Xueyun Zheng ${ }^{1}$, David E. Clemmer $^{2}$, Frank M. Raushel ${ }^{1}$, David H. Russell ${ }^{1}$, and Arthur Laganowsky ${ }^{1, *}$

${ }^{1}$ Department of Chemistry, Texas A\&M University, College Station, Texas.

${ }^{2}$ Department of Chemistry, Indiana University, Bloomington, Indiana. 

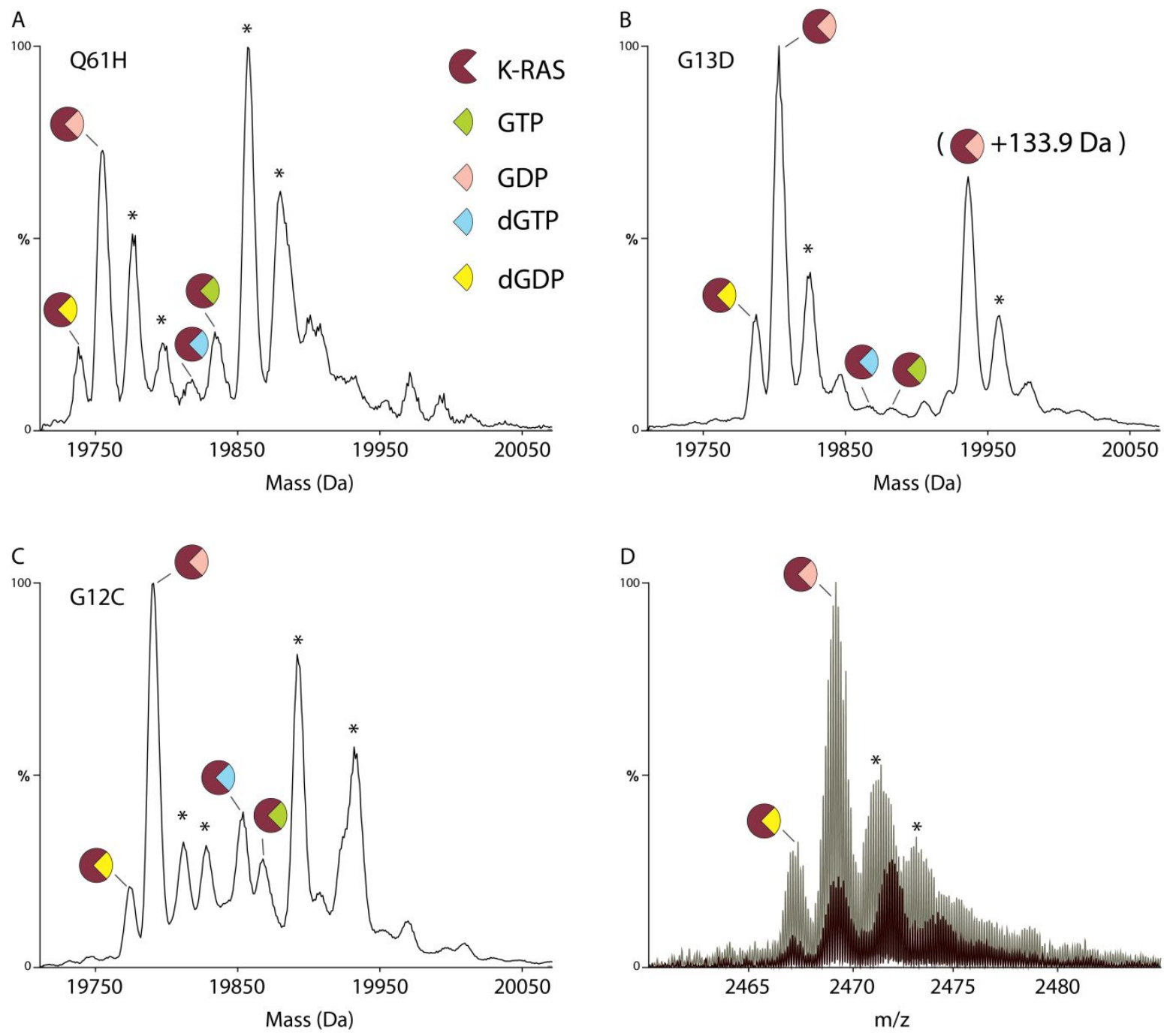

Figure S1. Deconvolution of native mass spectra for oncogenic K-RAS mutants (A) Q61H, (B) G13D and (C) G12C reveal binding of 2'-deoxy and 2'-hydroxy forms of GDP and GTP. (D) Overlay of native mass spectra of K-RAS (8+) as isolated (gray) and with magnesium acetate added to the spray solution (maroon). Asterisk denotes bound sodium or magnesium adducts. 
A

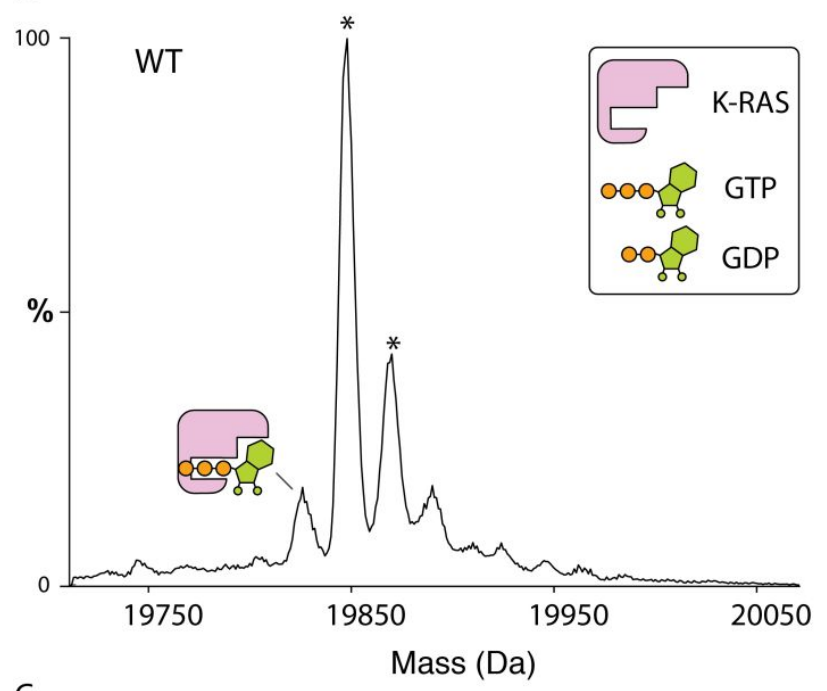

C

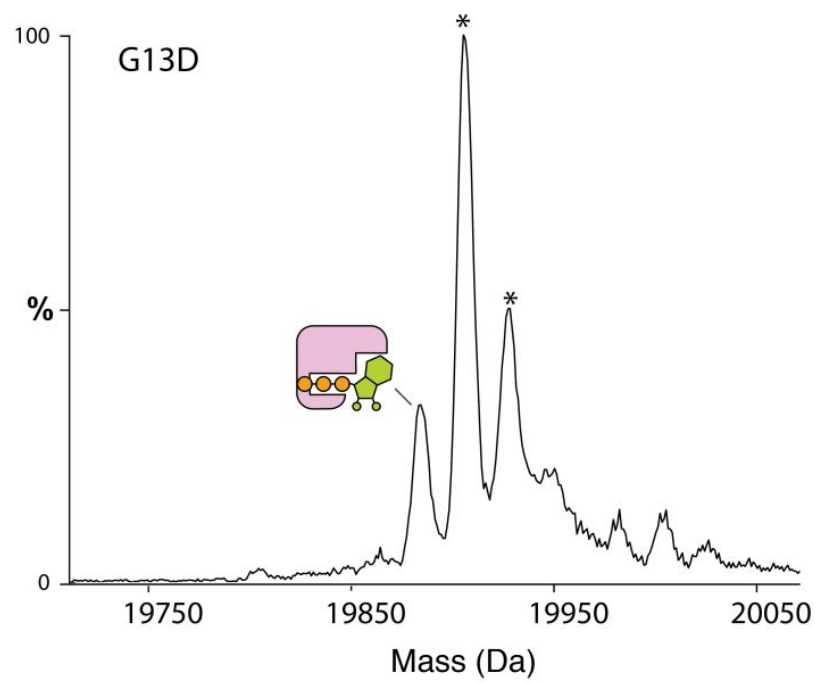

B

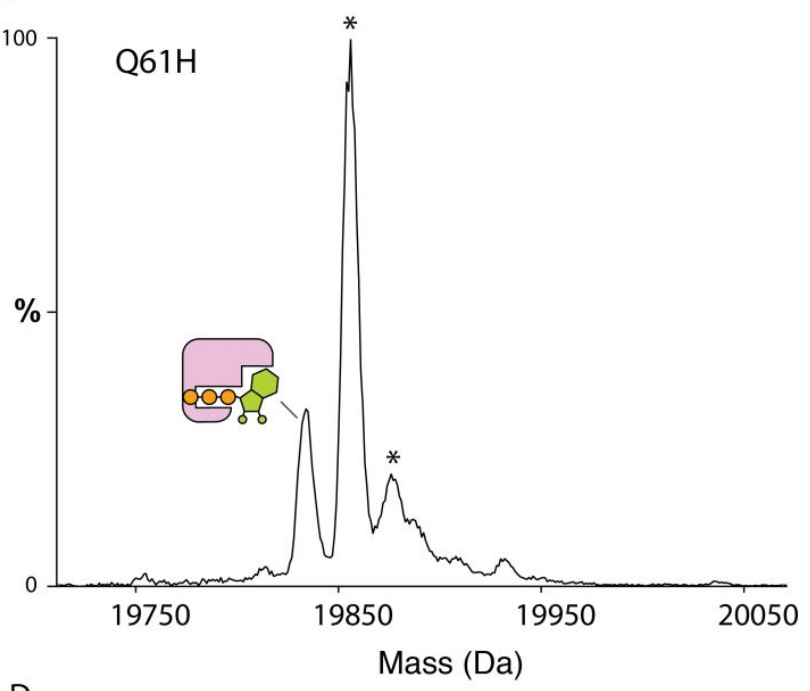

D

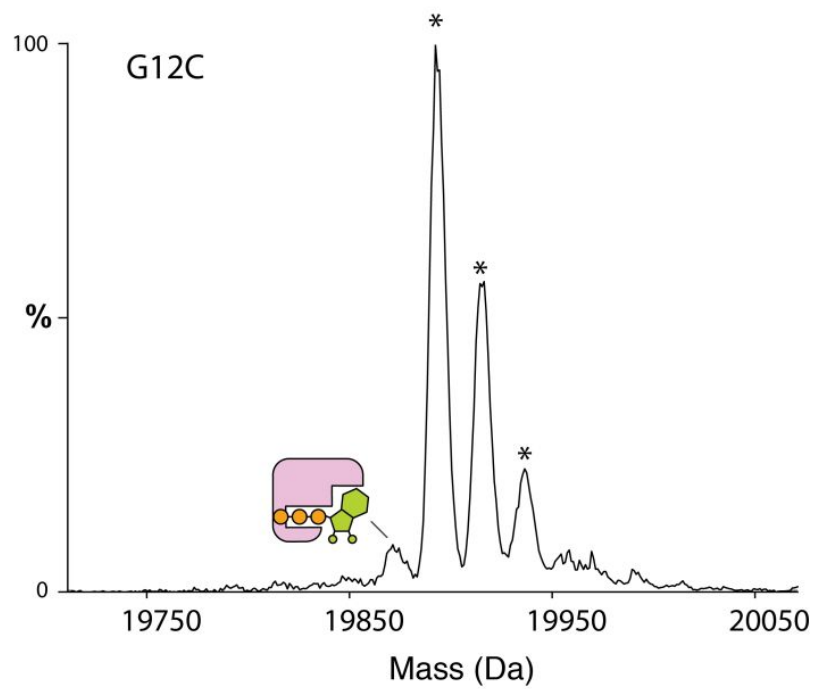

Figure S2. Deconvolution of native mass spectra for K-RAS (A) WT, (B) Q61H, (C) G13D and (D) G12C loaded with GTP. Asterisk represents bound sodium or magnesium adducts. 
A

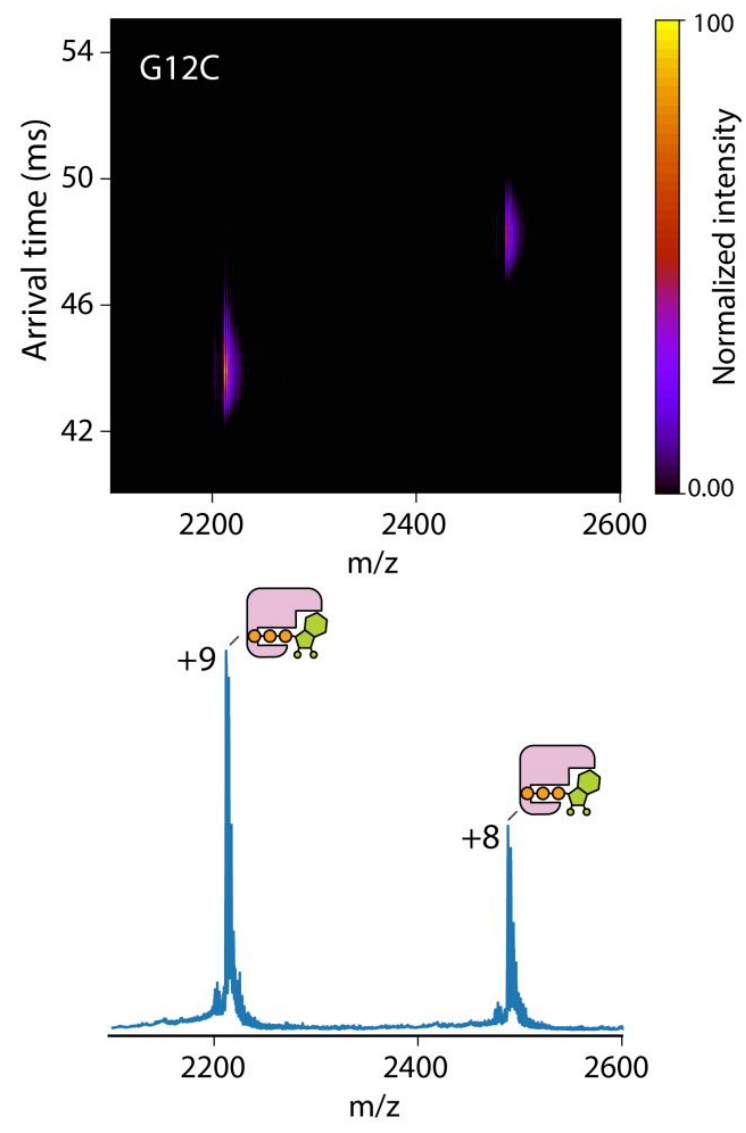

B

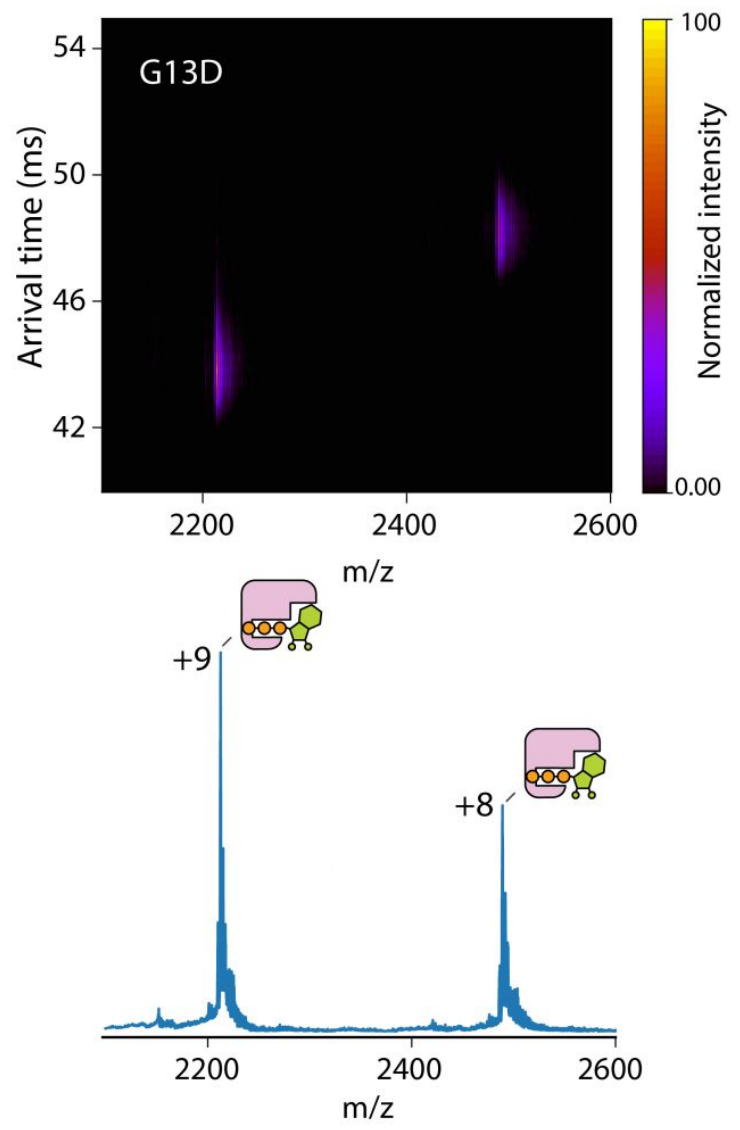

Figure S3. 2D Ion mobility mass spectra of K-RAS (A) G12C and (B) G13D. All mutants were loaded with GTP. 
A

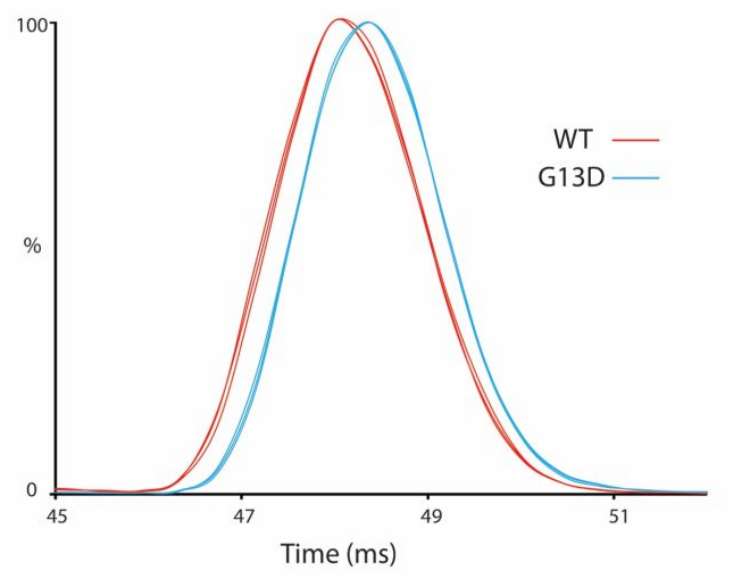

C

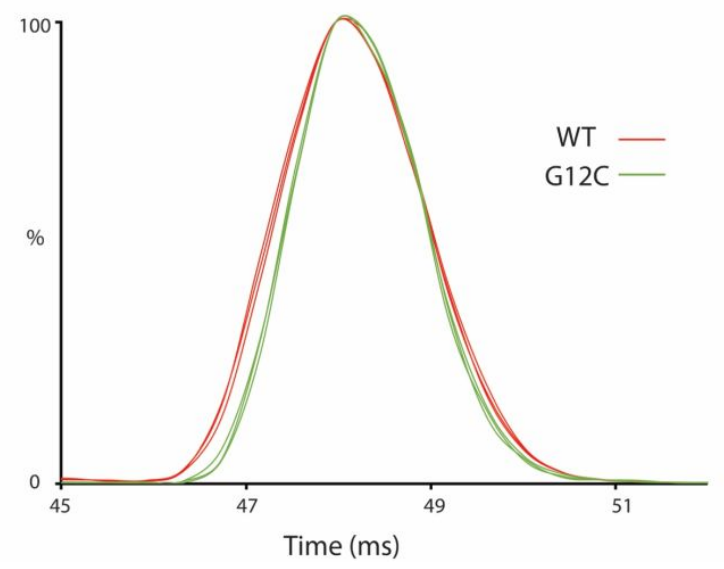

B

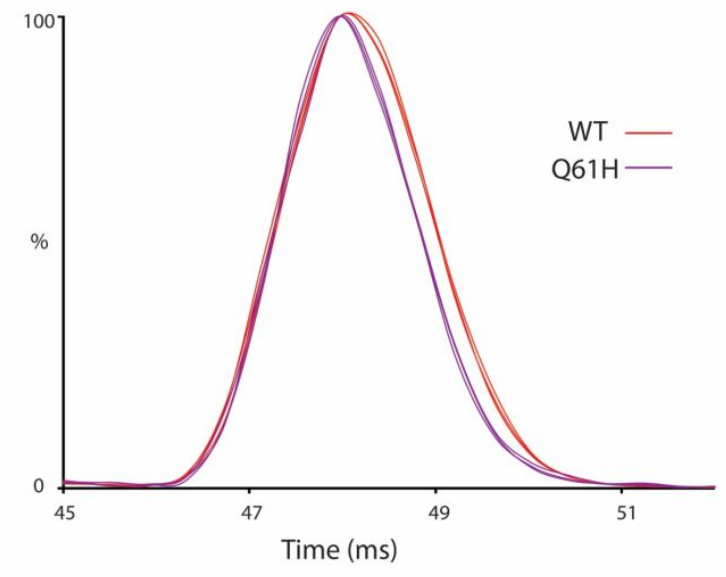

Figure S4. Comparison of arrival time distributions (ATD) of K-RAS and mutants bound to GTP. ATD are shown for the $8^{+}$charge state and repeated measurements overlaid. 

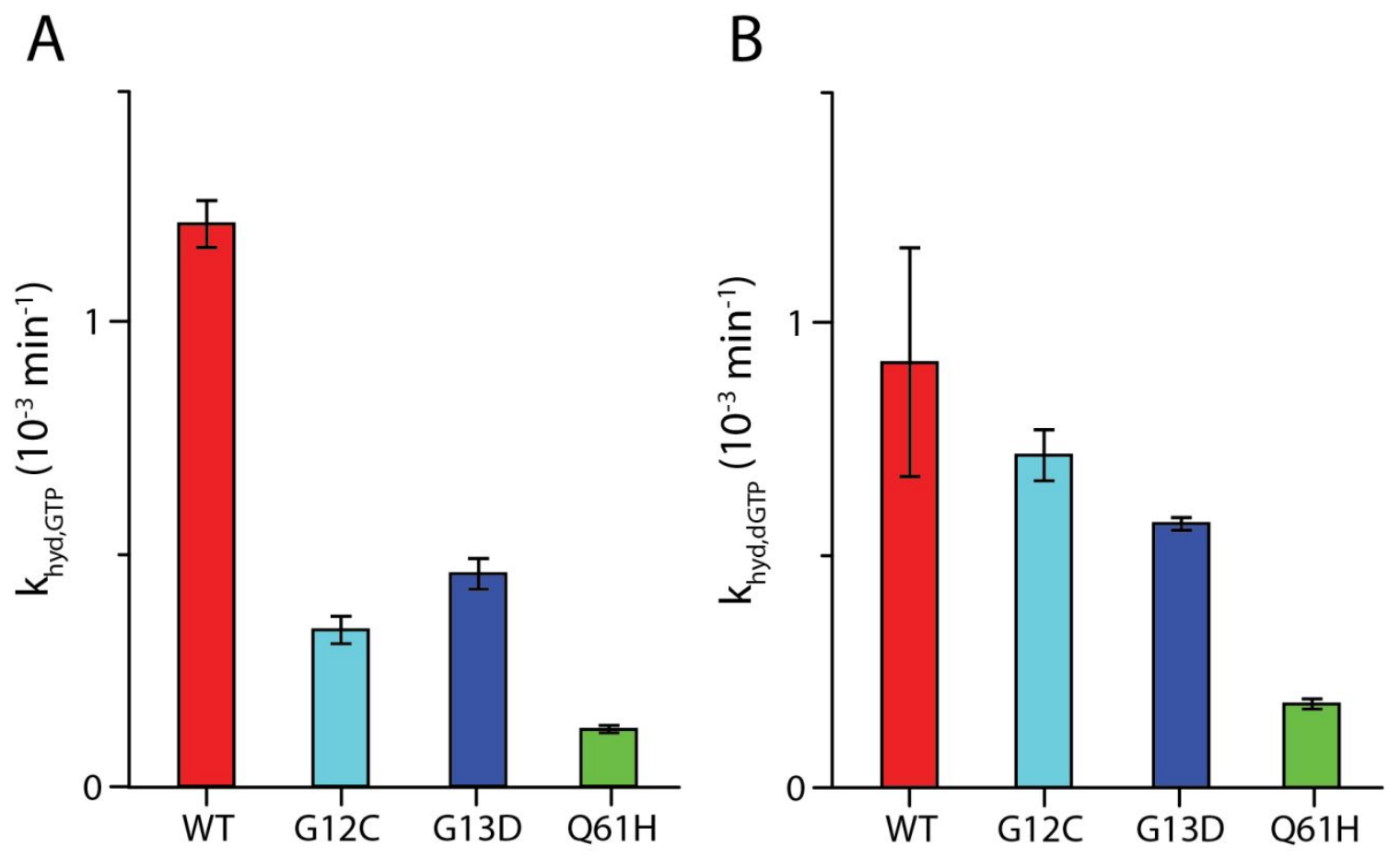

Figure S5. The rate of intrinsic GTP hydrolysis for K-RAS and mutants loaded with (A) GTP or (B) dGTP. Reported are the mean and standard deviation $(\mathrm{n}=3)$. 
A

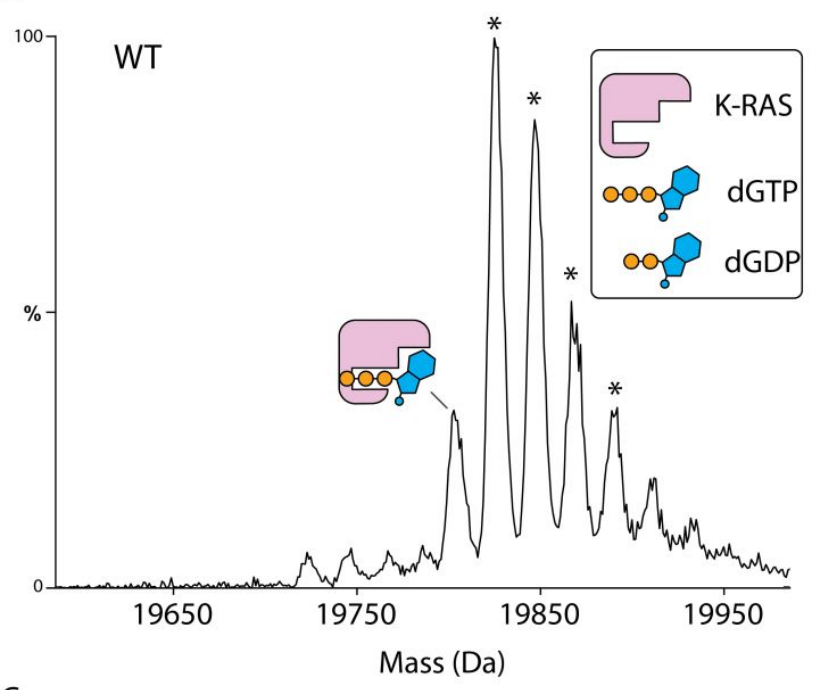

C

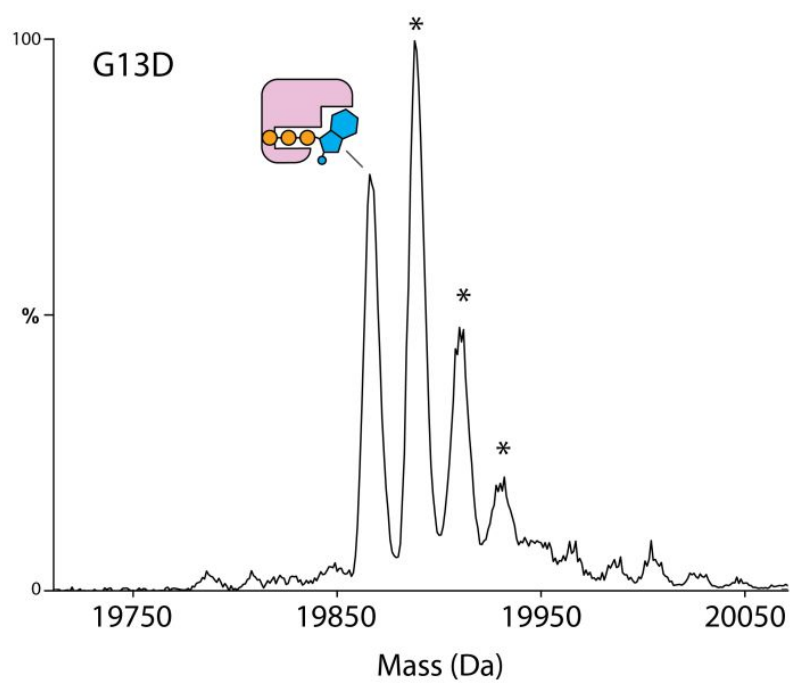

B
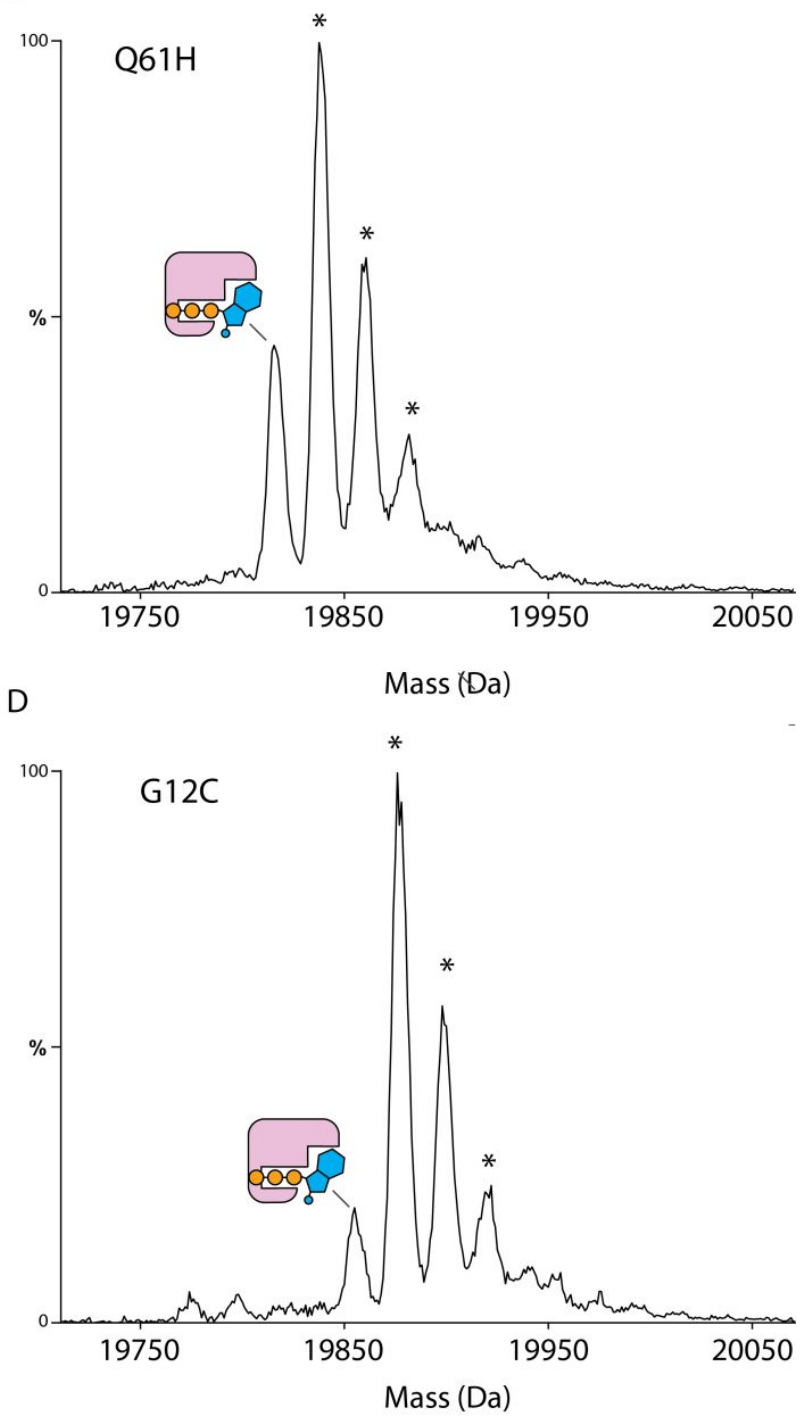

Figure S6. Deconvolution of native mass spectra for K-RAS (A) WT, (B) Q61H, (C) G13D and (D) G12C loaded with dGTP. Asterisk represents sodium or magnesium bound adducts. 
A
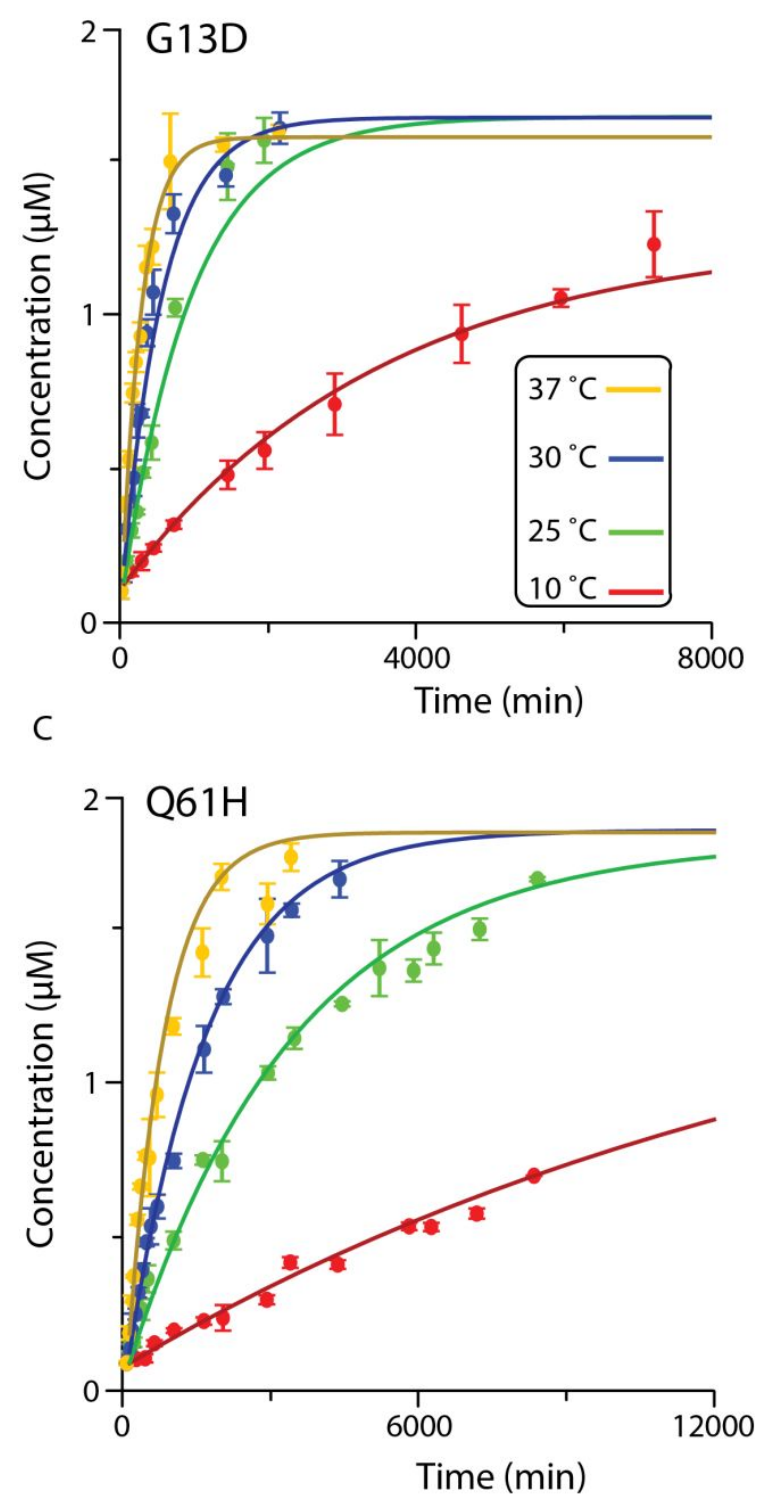

B

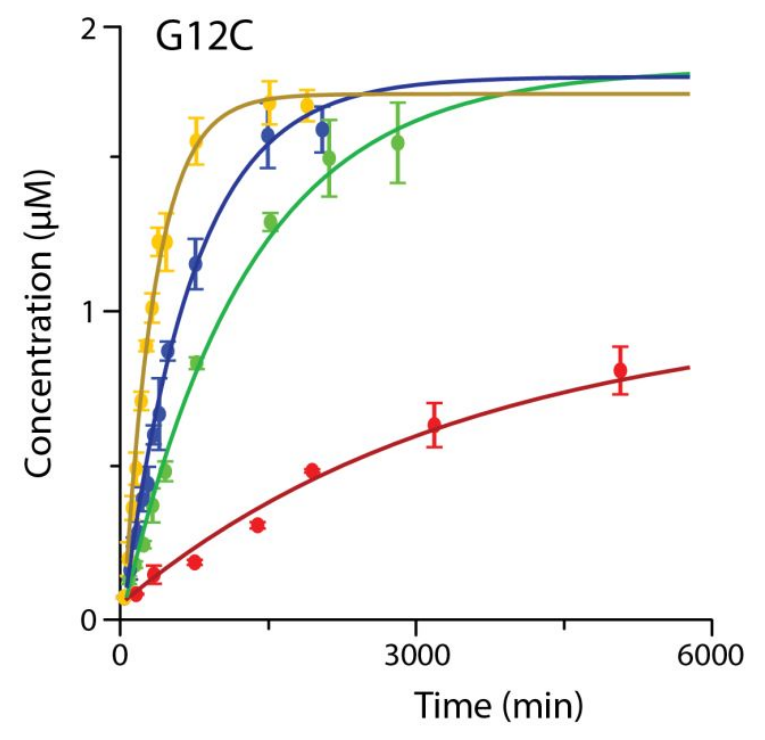

Figure S7. The temperature dependence of intrinsic GTP hydrolysis of K-RAS (A) G13D, (B) G12C and (C) Q61H. 

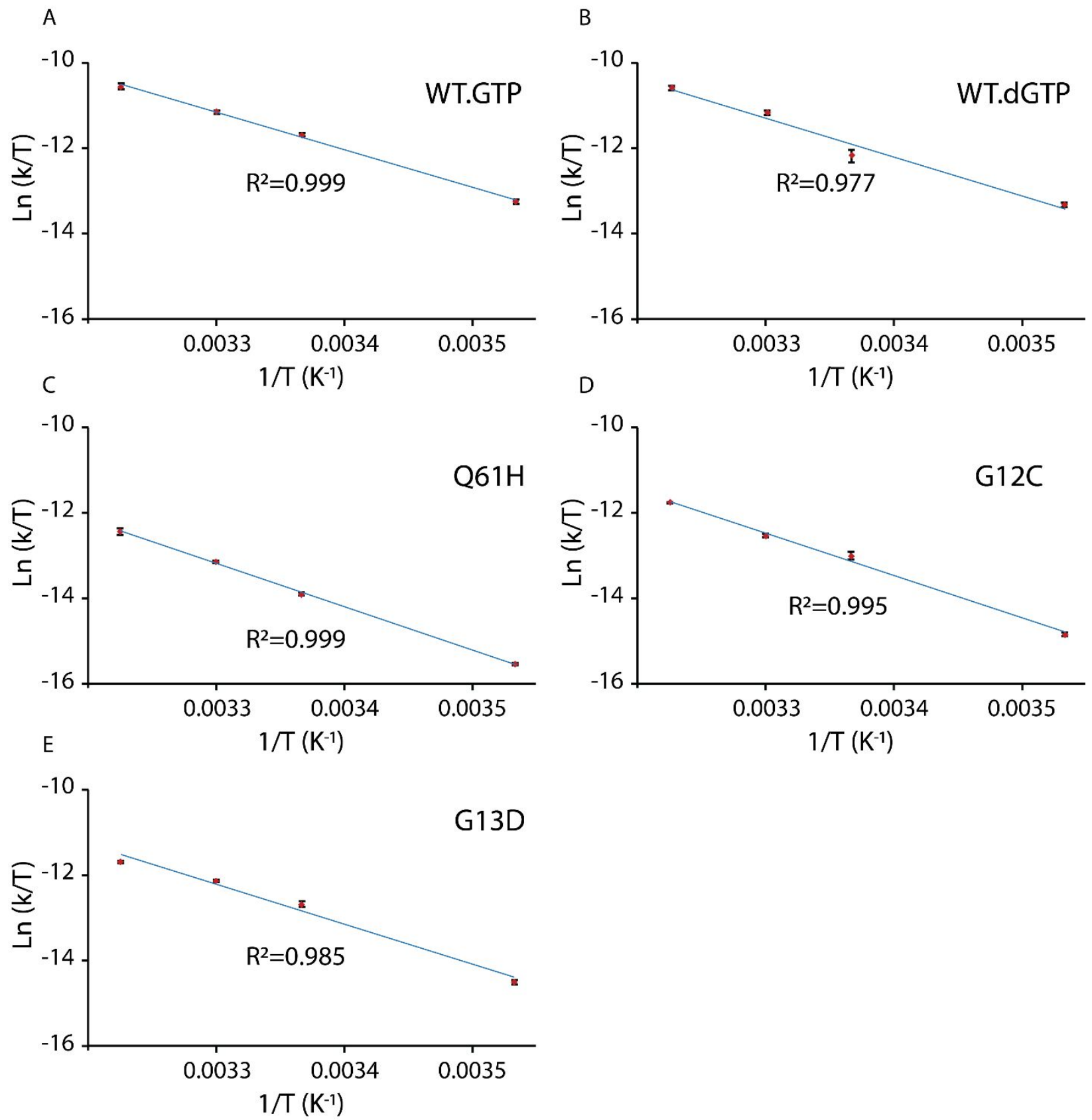

Figure S8. Eyring plots generated for K-RAS (A) WT•GTP, (B) WT•dGTP, (C) Q61H•GTP, (D) G12C•GTP and (E) G13D•GTP. All data shown are the mean and standard deviation $(n=3)$. 


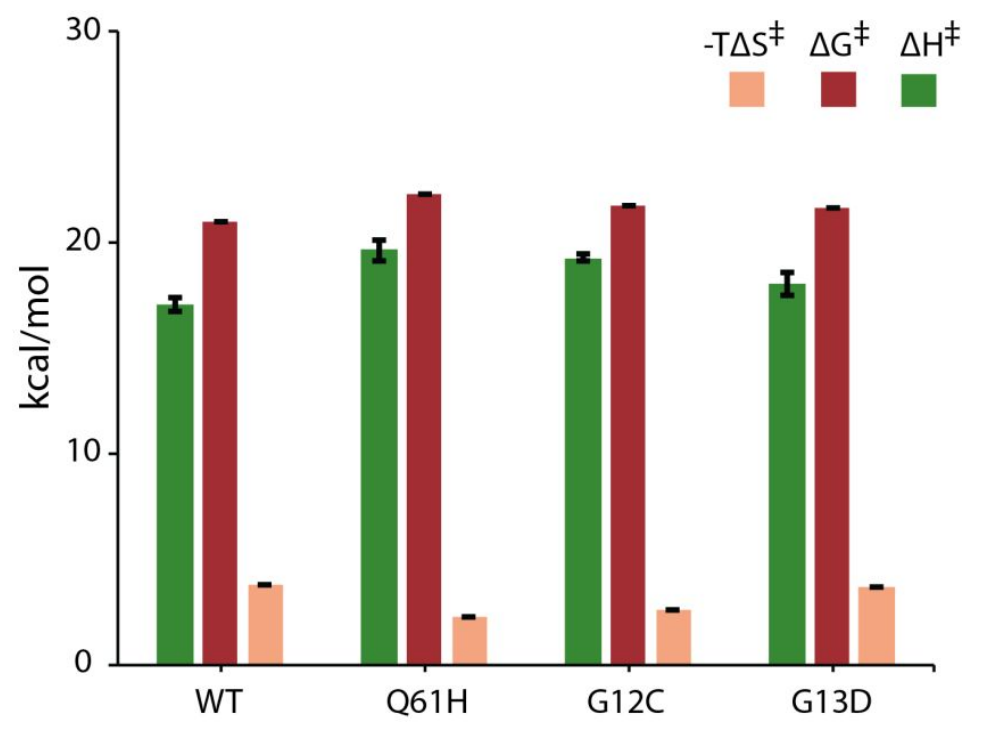

Fig S9. Enthalpy $\left(\Delta \mathrm{H}^{\ddagger}\right)$, entropy $\left(\Delta \mathrm{S}^{\ddagger}\right)$ and change in Gibbs free energy $\left(\Delta \mathrm{G}^{\ddagger}\right)$ of activation determined by Eyring analysis $(\mathrm{T}=298 \mathrm{~K})$. Reported are the mean and standard deviation $(n=3)$.

Table S1. The optimized parameters used for quantitative analysis of intrinsic hydrolysis using Q Exactive EMR.

\begin{tabular}{|l|c|}
\hline $\mathrm{m} / \mathrm{z}$ range & $2000-3000$ \\
\hline Resolution & 140000 \\
\hline In-source collision energy dissociation $(\mathrm{V})$ & 10 \\
\hline Source temperature $\left({ }^{\circ} \mathrm{C}\right)$ & 100 \\
\hline Capillary voltage $(\mathrm{KV})$ & 1.6 \\
\hline Source DC offset $(\mathrm{V})$ & 25 \\
\hline Injection flatapole lens $(\mathrm{V})$ & 15 \\
\hline Inter flatapole DC $(\mathrm{V})$ & 10 \\
\hline Bent flatapole DC $(\mathrm{V})$ & 6 \\
\hline Transfer multipole DC $(\mathrm{V})$ & 2 \\
\hline Pressure (mbar) & $3.4 \times 10^{-10}$ \\
\hline
\end{tabular}


Table S2. NanoESI condition and front funnel settings for the $20,000 \mathrm{~m} / \mathrm{z}$ extended mass range ion mode (Agilent 6560).

\begin{tabular}{|l|c|}
\hline Capillary voltage $(\mathrm{V})$ & $1000-2000$ \\
\hline Drying gas temperature $\left({ }^{\circ} \mathrm{C}\right)$ & 200 \\
\hline Drying gas flow $(\mathrm{L} / \mathrm{min})$ & 1.5 \\
\hline Fragmentor $(\mathrm{V})$ & 400 \\
\hline High Pressure funnel delta $(\mathrm{V})$ & 150 \\
\hline High Pressure Funnel RF $(\mathrm{V})$ & 200 \\
\hline Trap funnel delta $(\mathrm{V})$ & 180 \\
\hline Trap funnel RF $(\mathrm{V})$ & 200 \\
\hline Trap Funnel Exit $(\mathrm{V})$ & 10 \\
\hline
\end{tabular}

Table S3. Drift Tube Settings for Stepped Field Experiments in Positive Ion Mode

\begin{tabular}{|c|c|c|c|c|c|}
\hline Time Sequence & Time (min) & $\begin{array}{c}\text { Drift Tube } \\
\text { Entrance (V) }\end{array}$ & $\begin{array}{c}\text { Drift Tube Exit } \\
\text { (V) }\end{array}$ & $\begin{array}{c}\text { Rear Funnel } \\
\text { Entrance (V) }\end{array}$ & $\begin{array}{c}\text { Rear Funnel } \\
\text { Exit (V) }\end{array}$ \\
\hline 1 & $0.0-0.5$ & 1074 & 224 & 217.5 & 45 \\
\hline 2 & $0.5-1.0$ & 1174 & 224 & 217.5 & 45 \\
\hline 3 & $1.0-1.5$ & 1274 & 224 & 217.5 & 45 \\
\hline 4 & $1.5-2.0$ & 1374 & 224 & 217.5 & 45 \\
\hline 5 & $2.0-2.5$ & 1474 & 224 & 217.5 & 45 \\
\hline 6 & $2.5-3.0$ & 1574 & 224 & 217.5 & 45 \\
\hline 7 & $3.0-3.5$ & 1674 & 224 & 217.5 & 45 \\
\hline
\end{tabular}


Table S4. Theoretical and measured monoisotopic masses for 2'-deoxy and 2'-hydroxy forms of GDP and GTP bound to K-RAS WT and mutants. The measured monoisotopic mass is calculated by deconvolution the native mass spectra using Thermo protein deconvolution software.

\begin{tabular}{|cccccc|}
\hline Protein & Species & $\begin{array}{c}\text { Measured } \\
\text { monoisotopic } \\
\text { mass (Da) }\end{array}$ & $\begin{array}{c}\text { Theoretical } \\
\text { monoisotopic } \\
\text { mass (Da) }\end{array}$ & $\begin{array}{c}\text { Average mass } \\
\text { (Da) }\end{array}$ & ppm \\
\hline \multirow{2}{*}{ WT } & dGDP & 19717.69 & 19717.67 & 19729.12 & 0.938 \\
& GDP & 19733.71 & 19733.67 & 19745.04 & 2.11 \\
& dGTP & 19796.60 & 19796.63 & 19797.92 & 1.58 \\
\hline \multirow{2}{*}{ G12C } & GTP & 19810.56 & 19812.63 & 19820.96 & 104.57 \\
& dGDP & 19762.93 & 19763.64 & 19775.04 & 35.86 \\
& GGPP & 19778.96 & 19779.64 & 19791.04 & 34.40 \\
\hline \multirow{2}{*}{ G13D } & 19841.87 & 19842.60 & 19853.92 & 37.09 \\
& GTP & 19855.88 & 19858.60 & 19868.00 & 137.31 \\
\hline \multirow{2}{*}{ Q61H } & GGDP & 19775.69 & 19776.68 & 19786.72 & 50.04 \\
& GGTP & 19791.71 & 19792.68 & 19803.68 & 48.95 \\
& GTP & 19854.61 & 19855.64 & 19865.60 & 51.98 \\
& dGTP & 19873.57 & 19871.64 & 19881.60 & 97.42 \\
\hline & GTP & 19821.58 & 19822.63 & 19834.64 & 53.12 \\
\hline
\end{tabular}


Table S5. Collision cross section (CCS) values for the $8+$ charge state of K-RAS and mutants bound to dGTP. Reported are the mean and standard deviation for the centroid CCS values $(\mathrm{n}=$ $3)$.

\begin{tabular}{|c|c|}
\hline Protein & CCS $\left(\AA^{2}\right)$ \\
\hline K-RAS & $1937.6 \pm 10.9$ \\
\hline K-RAS $^{\mathrm{G} 12 \mathrm{C}}$ & $1936.2 \pm 7.4$ \\
\hline K-RAS $^{\mathrm{G} 13 \mathrm{D}}$ & $1941.0 \pm 5.2$ \\
\hline K-RAS $^{\mathrm{Q} 61 \mathrm{H}}$ & $1921.9 \pm 3.4$ \\
\hline
\end{tabular}

Table S6. The CCS calculated using IMPACT.

\begin{tabular}{|c|c|c|}
\hline Protein & PDB code & $\mathbf{C C S}\left(\mathbf{\AA}^{\mathbf{2}}\right)$ \\
\hline KRAS $^{\mathbf{W T}}$.GTP & 5VQ2 & $1909.4 \pm 6.3$ \\
\hline KRAS $^{\mathbf{Q 6 1 H}}$.GTP & 3GFT & $1924.7 \pm 7.9$ \\
\hline KRAS $^{\mathbf{W T}}$.GDP & 4OBE & $1948.3 \pm 5.1$ \\
\hline KRAS $^{\mathbf{W T}}$.GDP & 4LPK & $1822.4 \pm 3.9$ \\
\hline KRAS $^{\mathbf{G 1 2 C}}$.GDP & 4L8G & $1819.4 \pm 6$ \\
\hline KRAS $^{\mathbf{G 1 2 C}}$.GDP & 4LDJ & $2006.9 \pm 8$ \\
\hline KRAS $^{\mathbf{G 1 3 D}}$.GDP & 4TQA & $1952.9 \pm 7.2$ \\
\hline
\end{tabular}

Table S7. Thermodynamics of intrinsic GTPase activity for K-RAS loaded with dGTP.

\begin{tabular}{|c|c|c|c|}
\hline Protein & $\left.\Delta \mathbf{S}^{\ddagger} \mathbf{( k c a l} / \mathbf{m o l}\right)$ & $\Delta \mathbf{H}^{\ddagger}(\mathbf{k c a l} / \mathbf{m o l})$ & $\Delta \mathbf{G}^{\ddagger}(\mathbf{k c a l} / \mathbf{m o l})$ \\
\hline KRAS $^{\text {WT }}$.dGTP & $-0.0122 \pm 0.0009$ & $17.4 \pm 0.3$ & $21.06 \pm 0.04$ \\
\hline
\end{tabular}

